\title{
Pneumococcus a Rare Cause of Cellulitis
}

\author{
Muhammad U. Asghar ${ }^{1, *}$, Krishna Kommineni ${ }^{2}$, Sanwal S. Mehta ${ }^{2}$, Ravi Karan Patti ${ }^{2}$ \\ ${ }^{1}$ Pulmonary and Critical Care, Maimonides Medical Center, New York, USA \\ ${ }^{2}$ Internal Medicine, Maimonides Medical Center, New York, USA \\ *Corresponding author: Usmanjutt898@gmail.com
}

Received October 08, 2018; Revised November 29, 2018; Accepted December 28, 2018

\begin{abstract}
Streptococcus Pneumoniae (S. pneumoniae) is a common cause of community-acquired pneumonia, although its manifestation as a skin infection is a rare phenomenon. Most skin infections are secondary to Staphylococcus aureus or Streptococcus pyogenes, however, cellulitis caused by pneumococci is an infrequent finding. Pneumococcal cellulitis most often occurs due to bacteremia in patients with chronic illnesses such as diabetes, underlying malignancies, immunosuppressed patients, and patients with the history of injection drug abuse. The nature of the infection can be very fatal leading to fasciitis, myonecrosis, septic shock and ultimately death. We present a case of a 61-year-old female who developed painful swelling and redness of the right face along with bilateral leg involvement. In the emergency room, physical examination revealed multiple cervical lymphadenopathies. Further diagnostic investigations disclosed Low-grade B-cell non-Hodgkin lymphoma upon cervical lymph node biopsy. Blood culture grew Streptococcus pneumoniae as a source of cellulitis. This case demonstrates the importance of immune defects and the ensuing development of pneumococcal-induced cellulitis. Physicians should be vigilant while treating Pneumococcal cellulitis as this may be a sign of the serious underlying medical condition.
\end{abstract}

Keywords: Streptococcus pneumonia, cellulitis

Cite This Article: Muhammad U. Asghar, Krishna Kommineni, Sanwal S. Mehta, and Ravi Karan Patti, "Pneumococcus a Rare Cause of Cellulitis." American Journal of Medical Case Reports, vol. 6, no. 12 (2018): 247-248. doi: 10.12691/ajmcr-6-12-5.

\section{Introduction}

S. Pneumoniae is a gram-positive diplococcus; an infrequent cause of cellulitis involving face, neck, trunk and lower limbs. Pneumococcal Cellulitis is most commonly the result of bacteremia in patients with chronic illness such as diabetes, immunocompromised patients, injection drug users and patients with alcoholism [1,2]. Recently, Pneumococcal cellulitis is gaining more importance as these patients may have serious underlying disease pathologies such as malignancies. In cases of bacteremia; delayed treatment can cause fatal complications such as fasciitis, septic shock and may lead to death. Even with appropriate antimicrobial therapy many patients can undergo a prolonged hospital course and require advanced management. We present a case of a 61-year-old female who developed painful swelling and redness of the right face along with bilateral leg involvement. She was later found to have Low-grade B-cell non-Hodgkin Lymphoma and blood culture grew Streptococcus Pneumoniae as the cause of cellulitis.

\section{Case Presentation}

A 61-year-old female of Haitian origin with a past medical history of hypertension and obesity started having progressive shortness of breath (SOB) over 2 months. She was being treated with hydrochlorothiazide $25 \mathrm{mg}$ daily. Despite treatment, her SOB progressed. She developed painful swelling and redness of her face as well as in the legs bilaterally two days prior to presenting in the Emergency Department (ED). On physical examination, she had a temperature of 100.4 Fahrenheit, blood pressure of $114 / 56$, heart rate $116 / \mathrm{min}$ and respiratory rate of $20 / \mathrm{min}$. Her right face and both legs were swollen, erythematous, edematous and were tender to touch. She had an associated cervical lymphadenopathy with bilateral submandibular multiple lymph nodes of up to $1 \mathrm{x} 1 \mathrm{~cm}$ in size. The chest was clear to auscultation.

Due to suspicious of pulmonary embolism CT angiography of the chest was performed which showed extensive right Paratracheal, right hilar, and multiple bilateral axillary lymphadenopathies. No signs of pleural effusion or pneumothorax were seen. Initially, with a diagnosis of cellulitis, blood samples were drawn for culture and sensitivity. She was given a dose of Clindamycin. Complete blood count with a differential showed a white count of $7600 / \mathrm{ul}$ with 20\% bands, $\mathrm{Hb} 11.7 \mathrm{~g} / \mathrm{dl}$, Hematocrit 34.8\%, Lymphocytes $8 \%$, Neutrophils $46 \%$, Atypical Lymphocytes $6 \%$ and $\mathrm{HbA} 1 \mathrm{c} 6.3 \%$. HIV test was also negative. The echocardiogram was done to evaluate the cardiac cause of SOB and it showed normal ejection fraction with normal left ventricular size and no vegetation. The possibility of deep vein thrombosis (DVT) was assessed with bilateral venous duplex ultrasound which also came back negative.

Due to multiple enlarged lymph node in her chest, she was further evaluated for probable malignancy. Her CT neck 
showed massive diffuse bilateral cervical lymphadenopathy and evidence of enlarged mediastinal lymph nodes; findings compatible with lymphoma, leukemia or possibly metastasis. Furthermore, CT abdomen and pelvis also revealed extensive retroperitoneal, mesenteric, pelvic, and inguinal lymphadenopathy. She was admitted for management of sepsis secondary to cellulitis. Treatment was started with Ceftriaxone and Vancomycin intravenously (IV). Her blood culture grew gram-positive cocci in pairs and chains within 20 hours and later the organism was identified as Streptococcus pneumoniae. The bacteria were found to be sensitive to Penicillin. Her symptoms improved over the course of 2 weeks during which she received IV antibiotics. Repeat blood cultures were negative. Interventional radiology guided cervical lymph node biopsy was taken during her management and disclosed Low-grade B-cell non-Hodgkin lymphoma consistent with marginal zone lymphoma. She was discharged with outpatient follow up with Hematology/Oncology.

\section{Discussion}

S. Pneumoniae is a gram-positive diplococcus known to cause community-acquired pneumonia and meningitis. It normally colonizes respiratory tract, sinuses and nasal cavities in healthy individuals but can lead to bacteremia in patients with a weak immune system such as elderly, children and immunocompromised patients $[1,3]$. Staphylococcus aureus or Streptococcus pyogenes are the more frequent cause of cellulitis when associated with trauma, surgery or contiguous spread [4]. Historically $\mathrm{H}$ influenza type $\mathrm{b}$ has been the most common cause of cellulitis followed by, Streptococcus pneumoniae in absence of trauma or contiguous spread [5]. Since the introduction of $\mathrm{H}$ influenza type b vaccine, S. Pneumoniae predominates in such cases. Pneumococcal cellulitis presents as localized erythema, brawny discoloration, edema and to more serious conditions of bullae formation and skin desquamation [3,6]. It is considered that toxin may contribute to the development of local tissue inflammation in S. Pneumoniae cellulitis [7]. Although pneumococcal cellulitis has many causes, $92 \%$ of reported cases were secondary to bacteremia, which can ultimately lead to fatal complications. Diabetics, alcoholics, and injection drug user are more likely to have cellulitis of lower extremities [3], while patients with malignancies and systematic lupus erythematosus are more likely to have cellulitis in the face, neck, and torso [6,8].

Patients with immune defects caused by chemotherapy or cancers may have inadequate antibody response against pneumococcal capsular polysaccharides which will increase the risk of serious pneumococcal infection $[9,10]$. Similarly, deficiencies in complement proteins or defect in complement activation can also lead to an increased risk of severe pneumococcal bacteremia. Although, our patient initially presented with signs and symptoms of cellulitis but extensive lab investigations revealed the B-Cell lymphoma as the source of immune dysfunction, predisposing the patient to pneumococcal cellulitis. Moreover, certain risk factor such as age $>65$ years, assisted ventilation, chronic illness, and parenteral nutrition can also contribute to the development of Pneumococcal infections [11]. Vaccination against pneumococci in patients with cancers or chronic diseases induces less immunogenicity as compared to healthy individuals. These patients remain vulnerable to $\mathrm{S}$. Pneumoniae infection despite receiving vaccinations. S. Pneumoniae cellulitis is usually treated with Penicillin. The emergence of drug-resistant pathogen has made the treatment more challenging especially in patients who are immunocompromised [12].

\section{Conclusion}

Physicians should have a high level of suspicion of any underlying malignancy or immunocompromised state when a patient presents with cellulitis secondary to pneumococcus.

\section{Acknowledgements}

Author(s) have no conflict of interest to disclose and no funding was received from any source to conduct this research.

\section{References}

[1] Hinnen RM, Trachtenbarg DE, Miller MA, Coon JJ. "Streptococcus pneumoniae cellulitis". IMJ Ill Med J. 1986; 170(2): 84-6.

[2] Lewis RJ, Richmond AS, McGrory JP. "Diplococcus pneumoniae cellulitis in drug addicts". JAMA. 1975; 232(1): 54-5.

[3] Lawler MT, Crowe HM, Quintiliani R. "Cellulitis due to Streptococcus pneumoniae: case report and review". Clin Infect Dis. 1992; 14(1): 247-50.

[4] Israele V, Nelson JD. "Periorbital and orbital cellulitis". Pediatr Infect Dis J. 1987; 6(4): 404-410.

[5] Powell KR, Kaplan SB, Hall CB, at el. "Periorbital cellulitis: clinical and laboratory findings in 146 episodes, including tear countercurrent immunoelectrophoresis in 89 episodes". Am J Dis Child. 1988; 142(8): 853-857.

[6] Varghese R, Melo JC, Chun C-H, Raff MJ. "Erysipelas-like syndrome caused by Streptococcus pneumoniae". South Med J. 1979; 72(6): 757-8.

[7] Peters NS, Eykyn SJ, Rudd AG. "Pneumococcal cellulitis: a rare manifestation of pneumococcaemia in adults". J Infect. 1989; 19(1): 57-9.

[8] Hill MD, Karsh J. "Invasive soft tissue infections with Streptococcus pneumoniae in patients with systemic lupus erythematosus: case report and review of the literature". Arthritis Rheum. 1997; 40(9): 1716-9.

[9] Folland D, Armstrong D, Seides S, Blevins A. "Pneumococcal bacteremia in patients with neoplastic disease". Cancer. 1974; 33(3): 845-849.

[10] Ekdahl K, Braconier JH, Svanborg C. "Impaired antibody response to pneumococcal capsular polysaccharides and phosphorylcholine in adult patients with a history of bacteremic pneumococcal infection". Clin Infect Dis. 1997; 25(3): 654-660.

[11] Farinas-Alvarez C, Farinas MC, at el. "Prognostic factors for pneumococcal bacteremia in a university hospital". Eur J Clin Microbiol Infect Dis. 2000; 19(10): 733-741.

[12] Souweine B, Mom T, Bret L, at el. "Cellulitis due to Streptococcus pneumoniae with diminished susceptibility to penicillin in an immunocompromised patient". Scand J Infect Dis. 1997; 29: 518-9. 